\title{
Die Schattenseiten der Online-Partizipation: Veränderungen des demokratischen Diskurses in und durch die sozialen Medien
}

WolfJ. Schünemann

Das sog. europäische Superwahljahr 2017 mit Parlaments- oder Präsidentschaftswahlen in den Niederlanden, Frankreich, Großbritannien, Deutschland und Österreich war von großen Sorgen um die Gefährdungen der Demokratie geprägt. Diese Sorgen waren mit der Digitalisierung im Allgemeinen und den Veränderungen des demokratischen Diskurses in und durch soziale Medien eng verbunden. Sie fanden vor dem Hintergrund einer tieferen gesellschaftlichen und politischen Verunsicherung statt, die insbesondere durch irritierende internationale politische Ereignisse aus dem Vorjahr - genannt seien die Wahl Donald Trumps zum US-amerikanischen Präsidenten im November 2016 und die Referendumsentscheidung der Briten für einen Austritt aus der Europäischen Union im Juni desselben Jahres - hervorgerufen worden war. Die Art und Weise, wie die Kampagnen und Debatten in beiden Ländern auch mittels digitaler Medien und vor allem sozialer Netzwerke geführt und mutmaßlich beeinflusst worden waren, hatten diffuse Ängste entstehen lassen. Aus dieser Stimmung heraus sind ähnliche Entwicklungen, einschließlich demokratiegefährdender Manipulationen, auch für die jeweiligen nationalen Wahlkämpfe erwartet worden. Tatsächlich ließen sich die missliebigen Phänomene digitaler Kommunikation darin zwar ebenfalls beobachten, die schlimmsten Befürchtungen bestätigten sich jedoch nicht. Allerdings führten die Wahlen in allen genannten Ländern (mit Ausnahme Großbritanniens) zu deutlichen Zugewinnen für rechtspopulistische Bewegungen. ${ }^{1}$

1 Niederlande: Partij voor de Vrijheid plus 3\% ggü. den Vorwahlen, Deutschland: Alternative für Deutschland plus 7,9\%, Österreich: 5,5\%. In Frankreich schaffte es der Front National mit Marine Le Pen in der ersten Runde der Präsidentschaftswahlen mit 21,3\% in die Stichwahlen. Hier unterlag sie dem Zentrumskandidaten mit 33,9\% der Stimmen, alle Zahlen von www.wikipedia.org (abgerufen am 4. Februar 2018). 
Inwieweit sich diese Zugewinne auch neuen Mediennutzungsgewohnheiten, neuen Möglichkeiten der Kampagnenkommunikation und dem digitalen Strukturwandel der Öffentlichkeit zuschreiben lassen, wird gründlich und differenziert zu untersuchen sein. In jedem Fall haben die Erfolge der extremen Rechten zu den Zweifeln an einem positiven Beitrag digitaler Kommunikationsmedien zur Entwicklung der Demokratie beigetragen. Abgesehen von ihrem besorgniserregenden Anlass, bergen die Zweifel ihrerseits das Potential für Veränderungen unserer medial vermittelten demokratischen Öffentlichkeit. Denn aus ihnen nähren sich teils weitreichende Forderungen nach Regulierung von Internetinhalten. Nicht von ungefähr haben diese in Deutschland im Wahlkampfjahr Fuß gefasst und sind in Regulierungsmaßnahmen wie das deutsche Netzwerkdurchsetzungsgesetz (kurz: NetzDG) gemündet. ${ }^{2}$ Die den Kern des Gesetzes bildende Auflage an Betreiber großer sozialer Netzwerke, offensichtlich illegale Inhalte in einem vorgegebenen Zeitraum von ihren Plattformen zu löschen, hat eine lebhafte Kontroverse in Deutschland hervorgerufen. ${ }^{3}$ Auch international hat das NetzDG für Aufsehen und Kritik gesorgt. Der Streit um das NetzDG offenbart, wie sich in Deutschland und andernorts ein neuer netzpolitischer Großkonflikt um die Internetfreiheit herausbildet. In dieser Auseinandersetzung sind die Veränderungen des demokratischen Diskurses unter den Eindrücken der Digitalisierung von zentraler Bedeutung.

In den folgenden Abschnitten möchte ich diese Veränderungen im Rahmen verschiedener Annäherungen theoretisch und empirisch bestimmen. Dazu erläutere ich zunächst die demokratischen Versprechen, die seit Beginn der Internetentwicklung mit dieser verbunden sind (1). Im Anschluss daran werden auch die entgegengesetzten Zweifel ausgeführt (2). Für die empirische Betrachtung stehen dann die Phänomene von Hass und Hetze im Fokus, wobei diese anhand des deutschen Bundestagswahlkampfs und

2 Gesetz zur Verbesserung der Rechtsdurchsetzung in sozialen Netzwerken (Netzwerkdurchsetzungsgesetz - NetzDG), Bundesgesetzblatt Jahrgang 2017 Teil I Nr. 61, ausgegeben zu Bonn am 7. September 2017.

3 Für Kritik am NetzDG s. z.B. Constanze Kurz, »NetzDG: Viel Kritik, aber keine schnelle Evaluierung «, (netzpolitik.org, 9. Januar 2018), https://netzpolitik.org/2018 /netzdg-viel-kritik-aber-keine-schnelle-evaluierung/ (abgerufen am 20. April 2018), sowie eine Ausarbeitung des Wissenschaftlichen Dienstes des Deutschen Bundestages: Deutscher Bundestag, Wissenschaftliche Dienste, "Ausarbeitung. Entwurf eines Netzwerkdurchsetzungsgestzes. Vereinbarkeit mit der Meinungsfreiheit" (WD10 - 3000 - 037/17, 12. Juni 2017), https://www.bundestag.de/blob/510514/eef b7cf92dee88ec74ce8e796e9bc25c/wd-10-037-17-pdf-data.pdf (abgerufen am 20. April 2018). 
der Rolle der Alternative für Deutschland (kurz: AfD) untersucht werden (3). Abschließend folgen eine Zusammenfassung und einige Anschlussüberlegungen und -fragen (4).

\section{Die demokratischen Versprechen der Internetentwicklung}

Das Internet selbst und die auf ihm basierenden Anwendungen haben aufgrund ihrer soziotechnischen Eigenschaften seit jeher Erwartungen positiver Effekte der Demokratieentwicklung und Demokratisierung geweckt. ${ }^{4}$ Gerade in Zeiten der Verunsicherung über angebliche postdemokratische Tendenzen und Ernüchterungen über repräsentativdemokratische Realitäten schienen internetbasierte Kommunikationsformen und die durch sie beförderte politische Partizipation umfassende und nachhaltige Abhilfe schaffen zu können. ${ }^{5}$ Denn - so ließe sich das grundlegende Versprechen der technologischen Entwicklung mit den Worten eines führenden Demokratietheoretikers fassen - das Internet verspricht "völlig neue Chancen der Selbstorganisation der Bürgergesellschaft «. ${ }^{6}$ Net Empowerment, so wird die Annahme bezeichnet, wonach das Individuum durch das Internet ertüchtigt würde, das politische Geschehen stärker wahrzunehmen und daran direkter zu partizipieren. ${ }^{7}$ Mediale Gatekeeper und politische Intermediäre müssten hinter dem vernetzten und gestärkten Individuum zurücktreten, so die Idee. Dieses abstrakte Versprechen hat sich über die Jahrzehnte der Entwicklung neuer Anwendungen erhalten und ist vereinfacht gesagt in drei Varianten hervorgetreten.

Die erste Variante ging mit der Reifung des Internets als Massenkommunikationsmittel einher. In ihr werden viele Anleihen an die noch weiter zurückreichenden revolutionären Erwartungen der Entwickler-Communi-

4 Glenn Greenwald, »NSA: Die Schere im Kopf: Wie Massenüberwachung jeden Protest im Keim erstickt«, (2014) 6 Blätter für deutsche und internationale Politik 47, 47.

5 Marianne Kneuer, "Bereicherung oder Stressfaktor? Überlegungen zur Wirkung des Internets auf die Demokratie«, in Marianne Kneuer (Hrsg.), Das Internet: Bereicherung oder Stressfaktor für die Demokratie? (Nomos, 2013), 13.

6 Peter Graf Kielmansegg, Die Grammatik der Freiheit: Acht Versuche über den demokratischen Verfassungsstaat (Nomos, 2013), 262.

7 Alexander Siedschlag, Alexander Bilgeri und Dorothea Lamatsch, »Elektronische Demokratie und virtuelles Regieren: Erfahrungen und Perspektiven«, in Alexander Siedschlag, Alexander Bilgeri und Dorothea Lamatsch (Hrsg.), Kursbuch Internet und Politik: Elektronische Demokratie und virtuelles Regieren (Leske + Budrich, 2001), 10. 
ty gemacht. Im Wesentlichen geht es um die Umsetzung weitgehender partizipatorischer Visionen, wobei direktdemokratische Verfahren der Entscheidungsfindung, die bis dahin womöglich an den praktischen Zwängen gesellschaftlicher Organisationsfähigkeit in modernen Massengesellschaften gescheitert wären, im Computer- und dann im Internetzeitalter endlich umsetzbar schienen. ${ }^{8}$ Dahinter steht das antike Ideal der direkten Demokratie, ausgedrückt in dem Bild der Agora Athens. Passenderweise bemühte etwa der ehemalige US-amerikanische Vizepräsident Al Gore exakt dieses Bild in einer Rede vor der ITU in Buenos Aires 1994, um die politische Vision für das Zeitalter des Internet zu beschreiben. Die von ihm beschworene neue »Datenautobahn" sei geradezu eine "Metapher für die Demokratie«. Es werde ein "neues athenisches Zeitalter der Demokratie» kommen. ${ }^{9}$ Die Idee, eine womöglich dysfunktionale Herrschaftsform mit technischen Hilfsmitteln wieder ihrer ursprünglicheren Konzeption zuzuführen, verdichtete sich im Leitbild der digitalen Agora, auf der Entscheidungen für das Gemeinwesen besser und demokratischer gefällt werden könnten. Diese Hoffnung wurde auch in der wissenschaftlichen Debatte, vielfach mit denselben Bezügen zur Antike, artikuliert. ${ }^{10}$ Der Gedanke an eine technikgetriebene Verfassungsreform verfing in aller Regel nicht. Die Vorstellung, die Internettechnologie für mehr oder weniger weitreichende Formen der Bürgerbeteiligung zu nutzen, ist indes in Form zahlreicher demokratischer Innovationen elektronischer Partizipation umgesetzt worden. ${ }^{11}$

Die zweite Variante demokratischer Versprechen ist im Zuge einer weiteren Entwicklungsstufe des Internets aufgekommen. Die Anwendungen des sog. Web 2.0 oder Mitmachnetzes brachten einen Paradigmenwechsel. Allgemein wurde in dieser Zeit der Auftritt der produser zelebriert, also der Nutzer, die zugleich Produzenten von Inhalten sind, womit sie die klassischen Gatekeeper in den etablierten Medien, aber auch in der Politik

8 Benjamin R. Barber, Starke Demokratie: Über die Teilhabe am Politischen (RotbuchVerlag, 1994).

9 Zitiert nach Hubertus Buchstein, »Bittere Bytes: Cyberbürger und Demokratietheorie«, (1996) 4 Deutsche Zeitschrift für Philosophie 583, 585.

10 Maria H. Dettenhofer, "Eine neue Chance für die Basisdemokratie? Denkanstöße aus dem klassischen Athen", in Siedschlag, Bilgeri und Lamatsch (Hrsg.), Kursbuch Internet und Politik (Fn. 7), 93; Howard Rheingold, Virtuelle Gemeinschaft: Soziale Beziehungen im Zeitalter des Computers (Addison-Wesley, 1994).

11 Norbert Kersting, »The Future of Electronic Democracy«, in Norbert Kersting (Hrsg.), Electronic Democracy (Budrich, 2012), 11; Marianne Kneuer und Sebastian Harnisch, »Diffusion of E-Government and E-Participation in Democracies and Autocracies«, (2016) 4 Global Policy 548. 
zunehmend verdrängen würden. ${ }^{12}$ »Here comes everybody«, lautete der sprechende Titel des Buchs von Clay Shirky, mit dem er die freudvolle Stimmung und den epochalen Wandel vor Augen führte, der die elitengesteuerten Institutionen repräsentativer Demokratie fortan wesentlich herausfordern würde. ${ }^{13}$ Klassische Politakteure und mediale Gatekeeper würden künftig von jedermann, von den Grassroots oder Netroots vernetzter Gesellschaften infrage gestellt, ja potentiell ersetzt. ${ }^{14}$ Damit erschien das Festhalten an den etablierten Gatekeeper-Strukturen politischer Prozesse unzeitgemäß, und der Ausbau partizipatorischer Instrumente und Verfahren wurde zum Gebot der Stunde, nicht zuletzt auch um den Umbau von Politik und Verwaltung an den Aufmerksamkeits- und Nutzungsgewohnheiten der Bürger zu orientieren. Der Kampagnenmanager von Howard Dean, dem Kandidaten um die Präsidentschaftskandidatur der US-Demokraten 2004, bezeichnete das Internet unter dem Eindruck aufkommender sozialer Medien als "the most democratizing innovation we've ever seen, more so even than the printing press «. ${ }^{15}$ Abgesehen von der grundlegenden Euphorie, die in diesem Satz zum Ausdruck kommt, hat sich in dieser Zeit der Wahlkampf in und mit sozialen Netzwerken ausgesprochen gewandelt. Der Online-Wahlkampf ist zu einem dynamischen, sehr erfolgreichen und ertragreichen Geschäft und einer politischen Notwendigkeit geworden. Den Startschuss dafür bildete der US-Präsidentschaftswahlkampf 2008. Er löste in den Demokratien weltweit Begeisterung aus. Der Obama-Kampagne gelang erstmals nicht nur die Mobilisierung breiter Wählermassen über das social web, die zunächst relativ schwach ausgestattete Kampagne konnte über Crowdfunding auch Hunderte Mio. US-Dollar einwerben. Das setzte Trends für viele Demokratien. Bis heute gilt der Präsident-

12 Yochai Benkler, The Wealth of Networks: How Social Production Transforms Markets and Freedom (Yale University Press, 2006).

13 Clay Shirky, Here Comes Everybody: The Power of Organizing without Organizations (Penguin Books, 2008).

14 Axel Bruns, Blogs, Wikipedia, Second Life and Beyond: From Production to Produsage (Peter Lang, (2009); kritisch dazu: Matthew Scott Hindman, The Myth of Digital Democracy (Princeton University Press, 2009); Wolf J. Schünemann, »E-Government und Netzpolitik - eine konzeptionelle Einführung«, in Wolf J. Schünemann und Stefan Weiler (Hrsg.), E-Government und Netzpolitik im europäischen Vergleich (Nomos-Verlag, 2012), 9.

15 Zitiert nach: Hindman, The Myth of Digital Democracy (Fn. 14), 2. 
schaftswahlkampf 2008 als der Startschuss für den nunmehr unumgänglichen Online-Einsatz in Wahlkämpfen. ${ }^{16}$

Eine besondere Steigerung hat die Net-Empowerment-Hypothese in der dritten Variante demokratischer Versprechen erfahren. Denn mit ihr wurde das Internet regelrecht als »Befreiungstechnologie « verstanden. ${ }^{17}$ Diese Annahme erhielt vor einigen Jahren besonders große weltöffentliche Aufmerksamkeit. Die politischen und sozialen Erhebungen des so genannten Arabischen Frühlings boten eine dramatische Hintergrundfolie. Wurde das Internet seit jeher als Herausforderung für autokratische Regime weltweit betrachtet und seine Verbreitung gerade aus US-amerikanischer Sicht mit einer globalen Perspektive des Demokratieexports verknüpft, ${ }^{18}$ so wurde im Kontext des Arabischen Frühlings ganz konkret das befreiende Potential digitaler Kommunikationsmedien, allen voran sozialer Netzwerke, diskutiert. ${ }^{19}$

Wenn auch in unterschiedlicher Erscheinungsform hervorgetreten, so ist mit den verschiedenen Reinkarnationen des demokratischen Versprechens die grundlegende Annahme verbunden, dass die Internettechnologie den einzelnen Nutzer in den Stand versetze, sich von der Repräsentation oder Bevormundung durch Eliten, Gatekeeper und professionelle Instanzen zu emanzipieren. Gerade daraus erwüchsen Chancen für die Demokratieentwicklung. Genau dieser Zusammenhang wurde seit jeher auch bezweifelt. Im Folgenden wird es um frühe Infragestellungen der demokratischen Versprechen und ihre aktuell besonders kritische Lage gehen.

16 Simone Unger, »Online-Kampagnen im Bundestagswahlkampf 2009: Obama reloaded oder Experimente im Web 2.0?«, in Schünemann und Weiler (Hrsg.), EGovernment und Netzpolitik (Fn. 14), 375.

17 Kritisch dazu: Marianne Kneuer und Thomas Demmelhuber, »Die Bedeutung Neuer Medien für die Demokratieentwicklung: Überlegungen am Beispiel des Arabischen Frühlings«, (2012) 35 Informationen zur Politischen Bildung 30, 31.

18 Im Sinne des "Web of the Free«, Mark A. Shiffrin und Avi Silberschatz, »Web of the Free« New York Times (New York, 23. Oktober 2005).

19 Larry Diamond, »Liberation Technology«, (2012) 3 Journal of Democracy 69; Philip N. Howard und Muzammil M. Hussain, »The Upheavals in Egypt and Tunisia: The Role of Digital Media«, (2011) 3 Journal of Democracy 35; dazu kritisch: Kneuer und Demmelhuber, Die Bedeutung neuer Medien (Fn. 17). 


\section{Doch kein Lebenselixier für die Demokratie? - Elemente der Ernüchterung}

Gründe für Ernüchterung in Reaktion auf die demokratischen Versprechen gab es schon früh und wurden von verschiedenen Beobachtern hellsichtig formuliert. So erschien etwa im Jahr 1996 in der Deutschen Zeitschrift für Philosophie der Beitrag »Bittere Bytes: Cyberbürger und Demokratietheorie« von Hubertus Buchstein. Darin befasst sich der Autor kritisch mit der Internetentwicklung und macht in der damaligen internetbezogenen Demokratieforschung zwei Lager aus, die den demokratischen Versprechen in unterschiedlicher Weise anhingen: Optimisten und Neutralisten. Während Erstere die demokratieförderlichen Aspekte in der Internettechnologie selbst erblickten und somit die Einlösung der demokratischen Versprechen für unumgänglich erachteten, würden Letztere das Internet als neutrales Medium bewerten. Die Ertüchtigung des Einzelnen und die Stärkung der Demokratie seien aus dieser Perspektive nicht von alleine zu erwarten, würden aber durch geeignete Reformmaßnahmen durchaus erreicht werden können. ${ }^{20}$

Buchstein hält beiden Lagern entgegen, dass die für das politische Denken der Moderne grundlegende Differenzierung zwischen öffentlicher und privater Sphäre, zwischen Citoyen und Bourgeois durch die digitale, unmittelbare Kommunikation zunehmend brüchig, schlicht hinfällig werde. Personalisierte Newsfeeds und unvermittelte Kommunikation würden eine maßgeblich durch Medien vermittelte und aggregierte Öffentlichkeit sukzessive aushöhlen und schließlich ersetzen. ${ }^{21}$ Verschiedene Folgewirkungen dieser strukturellen Veränderungen würden auch das Funktionieren der Demokratie infrage stellen. Jedenfalls seien Zweifel an den wesentlichen Annahmen der Optimisten und Neutralisten begründet.

Konkret formuliert Buchstein insgesamt sechs Fragen bzw. Einwände, die er teils an die Optimisten, teils an Optimisten und Neutralisten richtet: 1. »soziopolitische Nebeneffekte der Internetentwicklung «, 2. »Exklusivität der Computerkultur «, 3. »die politische Ökonomie des Netzes«, 4. »Redefreiheit und demokratische Zensur«, 5. »Informationsüberfluss und demokratische Interaktion", 6. "Charakteristika des politischen Diskurses vor dem Bildschirm«. Ich werde diese zentralen, bereits vor mehr als zwanzig Jahren formulierten Einwände zunächst im Lichte aktueller Entwicklungen prüfen, bevor ich weitere Phänomene aus der heutigen Beobachtung ergänzen möchte.

20 Buchstein, »Bittere Bytes« (Fn. 9), 585.

21 Buchstein, »Bittere Bytes« (Fn. 9), 594. 
Die soziopolitischen Effekte ${ }^{22}$ berühren vor allem die Zukunft des Arbeitsmarkts und der Wertschöpfung. Sie stellen in der Tat heute - in Zeiten künstlicher Intelligenz und des Internets der Dinge - wesentliche gesellschaftliche Fragen des Digitalen Wandels dar. In einem jüngeren Werk hat etwa der Internettheoretiker Jaron Lanier die grundlegenden gesellschaftlichen Herausforderungen in diesem Zusammenhang herausgearbeitet und potentielle Lösungen entworfen. ${ }^{23} \mathrm{Da}$ die Frage einer zeitgemäßen und gerechten ökonomischen Ordnung für das digitale Zeitalter aber über das Kernthema dieses Beitrags, die Demokratieentwicklung, hinausgeht, wird dieser Aspekt hier übersprungen.

Die Exklusivität der Computerkultur, ${ }^{24}$ die Buchstein für Mitte der 1990er Jahre beklagt, ist für die demokratischen Versprechen des Internets weit gravierender, weil diese grundlegend infrage gestellt sind, wenn schon die Internetnutzung deutliche Asymmetrien aufweist, welche eine Ungleichverteilung von Einflussmöglichkeiten in der digitalen Demokratie verstärken oder zementieren würden. Nun sind die sog. digital divides in den meisten Industrienationen zurückgegangen. Es lassen sich aber immer noch Unterschiede nach Geschlecht, sozioökonomischem Status und Bildungsgrad, vor allem aber nach Alter feststellen. Die Abstände werden aber insgesamt geringer. ${ }^{25}$ Besonders auffällig sind demgegenüber weiterhin die sog. global digital divides, also solche Unterschiede, die sich zwischen verschiedenen Weltregionen, Ländern und Gesellschaften zeigen. ${ }^{26}$ Hier sind die langfristigen Differenzen der Teilhabemöglichkeiten immer noch erheblich. Für die hochentwickelten Staaten der industrialisierten Welt kann dennoch nicht mehr von einer Exklusivität der Computerkultur im Sinne Buchsteins gesprochen werden. Die Nutzung vernetzter Medien ist so alltäglich geworden und aus dem Alltag der meisten Menschen nicht mehr wegzudenken. Die Computer- oder besser: Digitalkultur ist damit universell geworden. Allerdings sind die Kompetenzen, Anwendungen im Netz zu gestalten und die Abläufe im Netz bis zu den technischen Grundlagen zu durchschauen, gewiss weiterhin einer technikaffinen Com-

22 Ebd., 589-590.

23 Jaron Lanier, Who Owns the Future? (Simon \& Schuster, 2014).

24 Buchstein, »Bittere Bytes« (Fn. 9), 590-592.

25 S. für Deutschland etwa die Ergebnisse des sog. Digital-Index (früher (N)OnlinerAtlas), Initiative D21, »D21-Digital-Index 2016« (2016), http://initiatived21.de/pu blikationen/d21-digital-index-2016/ (abgerufen am 4. Februar 2018).

26 Sebastian Harnisch und Wolf J. Schünemann, "Schlecht vernetzt ist halb verloren: Marktplatz der Ideen« (2016) 8 Ruperto Carola 51. 
munity vorbehalten, die keineswegs alle gesellschaftlichen Teilgruppen repräsentiert.

Die Befürchtungen, die Buchstein unter dem Titel »Mediapoly: Die politische Ökonomie des Netzes" anspricht, ${ }^{27}$ haben sich indes in vermutlich weit größerem Maße erfüllt, als vom Autor und anderen vorhergesehen. Das Internet hat dem zentralisierenden Druck der Anbieter und der Netzwerkeffekte nicht standgehalten. Die prinzipielle Offenheit der Internetarchitektur ist durch die erfolgreichen Internetunternehmen selbst, Diensteund Inhalteanbieter, zunehmend infrage gestellt worden. Das offene Netz, das frühere Generationen zu deliberativen Visionen bewegt hat, ist heute allenfalls noch mit Blick auf die grundlegende Infrastruktur erkennbar. Auf der Anwendungsebene haben allerdings zentrale Plattformen das Internetgeschehen durch verführerische Abonnements oligopolisiert. Die wenigen marktbeherrschenden Plattformen (Google, Microsoft, Apple, Amazon und Facebook) verwalten die digitalen Existenzen von Milliarden von Nutzern, die immer weniger Anlass verspüren, sich aus ihren Gated Communities in das offene Netz zu begeben, um dort im Zweifel schlechtere, weil nicht-personalisierte Services zu erhalten. In Anlehnung an Adorno und Horkheimer formulierte Buchstein einst: »Die Mediengroßkonzerne der $>$ Kulturindustrie $[\ldots]$ tendieren heute mehr dazu, jeden Teil des Medienprozesses und jedes Organ des Medienkonsumenten zu erreichen ${ }^{28}$ Zwei Jahrzehnte später umfasst der Versorgungsanspruch der großen Internetkonzerne die Informationsversorgung und Kommunikation nahezu vollständig. Die neuerliche Aushöhlung des Prinzips der Netzneutralität, ${ }^{29}$ also des Prinzips der Gleichbehandlung aller Datenpakete im Internet, zugunsten schnellerer Datenübertragung für bestimmte Daten und Kunden ist dazu angetan, die Offenheit der Internetarchitektur weiter fundamental infrage zu stellen und Machtstrukturen zu verfestigen, die der Annahme einer Demokratisierung entgegenstehen und stattdessen die oligopolistischen Tendenzen verstärken.

27 Buchstein, »Bittere Bytes« (Fn. 9), 592-593.

28 Buchstein, »Bittere Bytes« (Fn. 9), 592.

29 Unter der Obama-Präsidentschaft haben sich die USA unter großem internationalen Aufsehen zum Prinzip der Netzneutralität bekannt. Die Nachfolgeregierung unter Präsident Trump ist von diesem Prozess abgerückt. Cecilia Kang, »F.C.C. Plans Net Neutrality Repeal in a Victory for Telecoms « New York Times (New York, 21. November 2017), www.nytimes.com/2017/11/21/technology/fcc-net-neu trality.html (abgerufen am 4. Februar 2018). 
Auch die Herausforderung, die Buchstein unter dem Titel Redefreiheit und sdemokratische Zensur beschreibt, ${ }^{30}$ also die grundlegende Frage, ob Gesellschaften als Preis für die demokratische Freiheit der Rede Aufrufe zur Gewalt gegen Personen und Gruppen, Hassparolen oder rassistisches Gedankengut ertragen müssten oder ob sie durch freiheitsbeschränkende $\mathrm{Maßnahmen} \mathrm{dagegen} \mathrm{vorgehen} \mathrm{dürfen,} \mathrm{ist} \mathrm{keineswegs} \mathrm{unwichtiger} \mathrm{gewor-}$ den und ist auch heute Kernfrage kontroverser Debatten, etwa über das deutsche Netzwerkdurchsetzungsgesetz. Wie dieses und ähnliche Maßnahmen in anderen Ländern zeigen, ist der Ausgang des Streits, der sich als Verunmöglichung »demokratischer Zensur" durch »technischen K.O. «1 früh andeutete, so einfach nicht zu bestimmen. Denn zum einen lässt sich auch heute durchaus, wenngleich in Abstufungen, zwischen einer eher öffentlichen (Twitter, auch öffentliche FB-Posts und -Kommentare) und einer privaten Netzkommunikation (Chatnachrichten, E-Mails) unterscheiden. Aufgrund ihrer intendierten Öffentlichkeit oder Privatheit kommen die von Buchstein problematisierten Verschlüsselungstechnologien ohnehin nur bei Letzterer zum Einsatz. ${ }^{32}$ Bei Ersterer scheint die zunehmende Plattformisierung wiederum von Vorteil, wie das NetzDG unter Beweis stellt, weil sie den staatlichen Behörden mit der Einbindung und Verpflichtung der Betreiber sozialer Netzwerke durchaus weitreichende praktische Mittel zur Content-Regulierung an die Hand gibt. Demokratische Zensur ist also auch heute technisch möglich. Ob sie, zumal in dieser Form, wünschenswert ist, steht auf einem anderen Blatt. Weiter unten werde ich zeigen, welche empirische Evidenz im Wahljahr 2017 den Befürwortern demokratischer Zensur in Deutschland Nahrung verschafft hat.

Unter der Überschrift `Geöffnete Knoten«: Informationsüberfluß und demokratische Interaktion nimmt Buchstein eine Herausforderung vorweg, die wir spätestens seit 2016 in besonderer Vehemenz diskutieren. ${ }^{33}$ Dabei handelt es sich um den der Fragmentierung der Öffentlichkeit und der Indivi-

30 Buchstein, »Bittere Bytes« (Fn. 9), 593 ff.

31 Ebd., 593.

32 Wenn diese Verschlüsselungsanstrengungen dann zu leichtfertig und fundamental etwa im Rahmen von Ermittlungstätigkeit ausgehebelt werden - wie mit dem aktuell diskutierten sog. "Staatstrojaner«, der, auf dem Gerät des Überwachten installiert, noch vor der Verschlüsselung Nachrichteninhalte überträgt, der Fall haben wir es mit einem Angriff auf die Privatsphäre im Sinne des Panoptismus und nicht auf die Redefreiheit zu tun. Patrick Beuth und Kai Biermann, "Dein trojanischer Freund und Helfer « Zeit Online (Hamburg, 22. Juni 2017), www.zeit. de/digital/datenschutz/2017-06/staatstrojaner-gesetz-bundestag-beschluss (abgerufen am 4. Februar 2018).

33 Buchstein, »Bittere Bytes« (Fn. 9), 595-600. 
dualisierung der Informationsversorgung geschuldeten Glaubwürdigkeitsverlust von Information und ihren Trägern. Mit den Gatekeepern demokratischer Öffentlichkeit, insbesondere den klassischen Medien, sind durch die Internetentwicklung in der Tat auch die im demokratischen Staat multiplen Instanzen politischer Informationsversorgung relativiert worden. Die irritierende Folge ist die Verbreitung von Desinformationen, Gerüchten, "alternativen Fakten «, ${ }^{34}$ wobei die digitalen Verbreitungsmedien anders etwa als die klassischen Medienunternehmen durch die Streuung falscher Inhalte bislang keinen Reputationsverlust befürchten mussten. In der Tat scheinen die jüngeren Entwicklungen diese Immunität partiell aufgehoben und auf der anderen Seite dazu geführt zu haben, dass journalistische Redaktionen ihre Rolle als professionelle Informationsversorger wieder selbstbewusster definieren. Autorschaft und redaktionelle Sorgfalt werden als Kriterien für glaubwürdige Information auf den verschiedenen Plattformen wieder stärker sichtbar gemacht. Dies scheint ein pfadabhängiger Schritt auf dem Weg, den Buchstein für dieses Problem vorschlägt: »die Etablierung neuer [Informations]Knoten im Netz« ${ }^{35}$

Den Höhepunkt seiner Kritik erreicht Buchstein, wenn er unter dem Abschnittstitel /Körperlose Diskurse: Der politische Diskurs vor dem Bildschirm die empirisch beobachtbare Verrohung der politischen Debatte online beschreibt, ${ }^{36}$ die ganz anders, als es die natürlich idealisierte Erzählung von der klassischen athenischen Agora wiedergibt, ganz und gar nicht durch die Orientierung an der Konsensfindung orientiert sei, sondern zu unversöhnlich vorgetragenem Konflikt und Polarisierung tendiere. So sei

bei politischen Online-Debatten häufig eine Polarisierung durch Teilnehmer beobachtbar, die extreme Positionen beziehen. Solche Debatten verfallen häufig und schnell in einen Austausch von Verwünschungen und

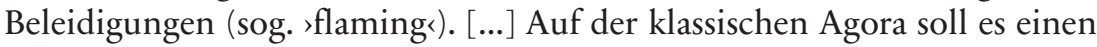
Wettstreit um die vernünftigste Art und Weise, ein Problem zu lösen und um den besten Weg zur Konsensbildung gegeben haben; im Wettstreit der elektronischen Agora hingegen gewinnt der haarsträubendste, provokanteste und verrückteste Standpunkt. Diese Einstellung macht die Entwicklung und Artikulation extremer politischer Positionen attraktiv.

34 So das berüchtigte Wort, das im Januar 2017 von einer Beraterin Donald Trumps gebraucht wurde, um die falschen Angaben des Pressesprechers Spicer zur Größe des Publikums bei der Amtseinführung des Präsidenten zu verteidigen. Die »alternativen Fakten« wurden 2017 in Deutschland und Österreich zum sog. »Unwort des Jahres« gewählt.

35 Buchstein, »Bittere Bytes« (Fn. 9), 599.

36 Ebd., 600-603. 
Man findet in der Kommunikation des Netzes viele überzeugt vorgetragene Meinungen, allerdings wenig informierten Dialog und vor allem kaum Dialoge, die an der Suche nach einem Konsens orientiert sind. ${ }^{37}$

Mag Buchstein an diesem Punkt auch zu einer überspitzten kulturkritischen Diagnose gelangen, so haben empirische Untersuchungen und kommunikative Alltagserfahrungen gezeigt, dass sog. flame wars in jedem Online-Forum sowie in den Kommentarbereichen sozialer Medien etc. eine natürliche Begleiterscheinung auch der politischen Kommunikation darstellen. In der unten beschriebenen eigenen Studie zur Online-Debatte während des deutschen Bundestagswahlkampfs 2017 finden sich insbesondere Anzeichen für die Verrohung von Sprache durch das verstärkte Auftreten von beleidigenden, hasserfüllten, unzivilisierten Äußerungen.

Über die Problembeschreibung hinaus, die Buchstein aus Sicht der Demokratietheorie bietet, lassen sich auch im Lichte der jüngeren Entwicklungen noch weitere Einwände gegen die demokratischen Versprechen des Internets zusammenfassen. Dies betrifft zunächst wesentliche Aspekte der grundlegenden Freiheit, die der Internetinfrastruktur zugeschrieben wird. Sie ist nur scheinbar gegeben. Tatsächlich ist das Internet "per se nicht demokratisch und kann aus sich heraus auch keine Demokratie erzeugen «.38 Die Internet Governance-Expertin Laura DeNardis hält unmissverständlich fest:

It is tempting to romanticize Internet architecture and governance as innately embodying democratic values of equality, participatory openness, and multistakeholder oversight but there are several problems with this narrative. In a significant portion of the world, Internet governance control structures do not embody democratic values but involve systems of repression, media censorship, and totalitarian surveillance of citizens. ${ }^{39}$

Der grundlegende Widerspruch gegen die Net-Empowerment-Hypothese ist in der sog. Verstärkerhypothese oder Reinforcement-These festgehalten. Wird partizipativen Anstrengungen generell die paradoxe empirische Beobachtung entgegengehalten, dass die neuen Verfahren und Mechanismen die Selektionseffekte in der tatsächlichen Beteiligung noch einmal erheblich verstärkten, so trifft das für die Partizipation über das Internet noch in gesteigertem Maße zu. Das ist der Grundgedanke der Verstärkerhypothese,

37 Ebd., 601.

38 Marianne Kneuer, Mehr Partizipation durch das Internet?, (LpB Rheinland-Pfalz, 2013), 14.

39 Laura DeNardis, The Global War for Internet Governance (Yale University Press, 2014), 15. 
die den demokratischen Versprechen und der Net-Empowerment-Annahme diametral entgegensteht. ${ }^{40}$ Für sie haben sich durchaus immer wieder Anzeichen in verschiedenen empirischen Studien finden lassen. So bestätigt Hindman die Annahme für die US-amerikanische Blogosphäre. ${ }^{41}$ Stier, Schünemann und Steiger kommen in einer politikfeldvergleichenden Studie der globalen Twitter-Kommunikation zu einem ähnlichen Ergebnis. ${ }^{42}$

Der politischen Beteiligung via Internet ist auch immer wieder ihre Kurzatmigkeit, mangelnde Ernsthaftigkeit und emotionale Erregtheit vorgehalten worden. Die politischen Ausdrucksformen online konzentrierten sich auf vergleichsweise oberflächlichen Slacktivism. Damit sind diese Formen der Internetnutzung also nicht einfach mit politischer Beteiligung gleichzusetzen, auch wenn es sich um im weitesten Sinne politische Inhalte handelt, die online konsumiert werden. ${ }^{43}$

The other end is a million people sitting at home alone, forming their opinion privately and expressing it directly by pushing a button, taking no responsibility for it, talking to no one about it, communicating with nobody and voting anonymously. ${ }^{4}$

Viele Formen der suggerierten Beteiligung im Internet sind mangels realistischer Erfolgsaussichten und gefangen in der sog. "Filterblase « 45 als

40 Siedschlag, Bilgeri und Lamatsch, »Elektronische Demokratie« (Fn. 7), 13.

41 Hindman, The Myth of Digital Democracy (Fn. 14), 4.

42 Sebastian Stier, Wolf J. Schünemann und Stefan Steiger, »Of Activists and Gatekeepers: Temporal and Structural Properties of Policy Networks on Twitter«, (2017) 2 New Media \& Society 1.

43 Vgl. Samuel Salzborn, Demokratie: Theorien, Formen, Entwicklungen (UTB, 2012), 113.

44 Benjamin R. Barber, »Which Technology for which Democracy? Which Democracy for which Technology? «, in Bernd Holznagel, Andreas Grünwald und Anika Hanssmann (Hrsg.), Elektronische Demokratie: Bürgerbeteiligung per Internet zwischen Wissenschaft und Praxis (Beck, 2001), 209, 216.

45 Der Begriff geht auf Eli Pariser zurück, Eli Pariser, The Filter Bubble: What the Internet is Hiding from You, (Penguin Press, 2011). Er hat in der öffentlichen Debatte eine große Wirkung erzielt. Ob die Mechanismen des "more of the same" in der Internetkommunikation tatsächlich gegeben sind oder ob die Nutzer nicht doch eher auf Unverhofftes und Unbekanntes stoßen als in der Offline-Kommunikation, ist eine Frage, die die Wissenschaft bewegt. Eindeutige empirische Befunde gibt es nicht. Viele Studien deuten aber auf eine Überbewertung des Phänomens durch die Kritiker, vgl. Aleksandra Sowa, Digital Politics. So verändert das Netz die Demokratie: 10 Wege aus der digitalen Unmündigkeit (Dietz, 2017), 23-25. 
bloße »Scheinpartizipation« zu bewerten. ${ }^{46}$ Beteiligung ist also nicht gleich Beteiligung - und das gilt in besonderem Maße für die Netzaktivitäten.

\section{Hate Speech im deutschen Bundestagswahlkampf}

Vor allem für sog. populistische Bewegungen, die sich als ungefilterte Stimme des Volkes inszenieren und oftmals mit der Vermittlung durch etablierte Medien, die sie als Teil des bekämpften Systems ansehen, hadern, sind soziale Medien von immenser Bedeutung. Nach einer Phase, in der die organisationslosen Koordinations- und Organisationsmöglichkeiten sozialer Bewegungen online überwiegend mit neuartigen Gruppierungen auf der politischen Linken (man denke an Occupy, Podemos o.a.) assoziiert worden waren, ${ }^{47}$ hat sich in jüngeren Jahren die Bedeutung der sog. connective action ${ }^{48}$ für die politische Rechte gezeigt. ${ }^{49} \mathrm{~W}$ ie oben belegt, gingen auch die oben genannten Wahlen überwiegend mit deutlichen $\mathrm{Zu}$ gewinnen für sog. rechtspopulistische Parteien aus. In Deutschland ist mit der Alternative für Deutschland, kurz AfD, sogar erstmals in der jüngeren Vergangenheit eine parteipolitische Formation dieser Art in den Bundestag eingezogen. Vor diesem Hintergrund untersuchen wir im Rahmen eines empirischen Forschungsprojekts, wie sich die AfD sozialer Medien bedient hat und welche Folgen dies für den demokratischen Diskurs in Deutschland hat. ${ }^{50}$ Konkret fragen wir, ob Hassbotschaften, Pöbeleien und Anfeindungen im Online-Wahlkampf 2017 eine gesteigerte Rolle gespielt haben und wie die Kommunikation von rechts außen in diesem Zusammenhang zu bewerten ist. Wir haben uns dabei vornehmlich auf Facebook als Datenquelle gestützt. Facebook ist weiterhin das soziale Netzwerk. Mögen sich jüngere Zielgruppen vermehrt auch anderen Plattformen zuwenden, erreicht kein Konkurrent eine vergleichbare Reichweite wie

46 Salzborn, Demokratie (Fn. 44), 115.

47 Marianne Kneuer und Saskia Richter, Soziale Medien in Protestbewegungen: Neue Wege für Diskurs, Organisation und Empörung? (Campus-Verlag, 2015).

48 W. Lance Bennett und Alexandra Segerberg, The Logic of Connective Action: Digital Media and the Personalization of Contentious Politics (Cambridge University Press 2013).

49 Vgl. u.a. Sebastian Stier et al., »When Populists Become Popular: Comparing Facebook Use by the Right-Wing Movement Pegida and German Political Parties«, (2017) Information, Communication \& Society 1365.

50 Das Forschungsprojekt "Wahlkampf in (a)sozialen Netzwerken« ( $\left.\mathrm{WasN}_{\mathrm{BTW}}{ }_{17}\right)$ wurde von einem Konsortium von Wissenschaftlerinnen und Wissenschaftlern an den Universitäten Hildesheim, Göttingen und Heidelberg durchgeführt. 
Facebook. Laut Reuters Digital News Report 2020 nutzen mittlerweile 49 Prozent der Deutschen Facebook; immerhin 22 Prozent der Befragten gaben an, Facebook auch für die Nachrichtenversorgung und Versorgung mit pol. Informationen zu verwenden. ${ }^{51}$

Abbildung 1: Aktivitätsverteilung (Meldungen und Kommentare) auf den Facebook-Seiten der Parteien ${ }^{52}$

700.000

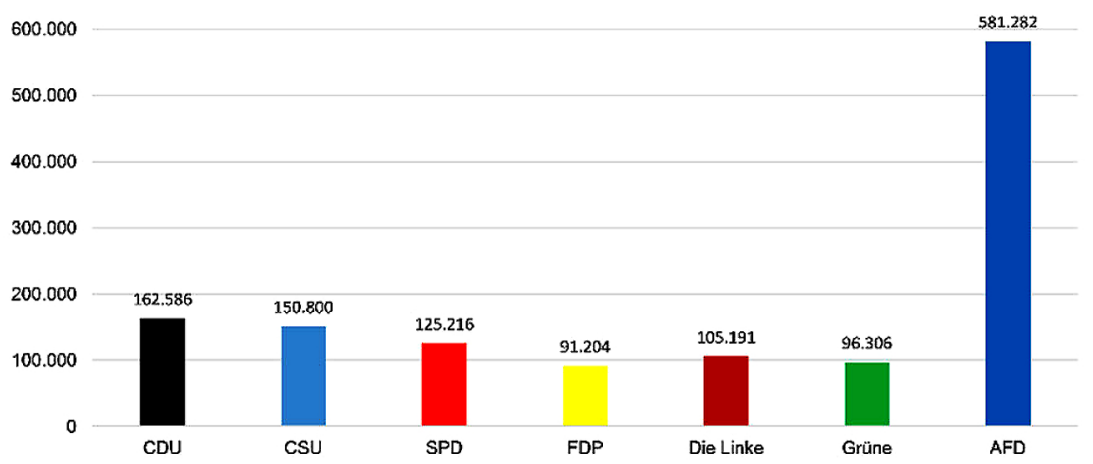

Für unsere Studie haben wir für einen mehrmonatigen Zeitraum im Vorfeld der Bundestagswahl 2017 die Aktivitäten auf den öffentlichen Facebook-Seiten der Parteien mit einer realistischen Aussicht auf Einzug in den Bundestag automatisch erhoben (unter Verwendung der Facebook Graph API). ${ }^{53}$ Dies waren im Einzelnen CDU, CSU, SPD, Bündnis 90/Die Grünen, FDP, Die Linke und die AfD. Das Gleiche haben wir für die Spitzenkandidatinnen und Spitzenkandidaten getan, sofern sie über entsprechende Facebook-Seiten verfügten. Zur Exploration und Analyse dieses großen Datensatzes (insg. etwa 2,9 Mio. Einträge) haben wir einfache statistische Operationen, korpuslinguistische Verfahren, ${ }^{54}$ wie die Schlüsselwort-Bildung und die Ermittlung von Kollokationen zum Einsatz gebracht. Explo-

51 Nic Newman et al., »Digital News Report 2020« Reuters Institute (Oxford, 2020), https://reutersinstitute.politics.ox.ac.uk/sites/default/files/2020-06/DNR_2020_FI NAL.pdf (abgerufen am 10. Juli 2020).

52 Quelle: eigene Darstellung.

53 Für die Definition des Untersuchungszeitraums haben wir die Ernennung des SPD-Kanzlerkandidaten am 29. Januar 2017 als Wahlkampfauftakt gewertet.

54 Für unsere korpuslinguistischen Arbeitsschritte verwenden wir das R Stylo-Paket sowie die Analyseplattform SketchEngine. 
rierte Befunde konnten in ihren jeweiligen Kontexten auch qualitativ untersucht werden. Auf dem knappen Raum dieses Beitrags können nur erste schlaglichtartige Befunde hinsichtlich Quantität und Qualität der politischen Kommunikationsaktivität der AfD im deutschen Bundestagswahlkampf präsentiert werden.

Tatsächlich ist der Alternative für Deutschland (AfD) im Bundestagswahlkampf eine beispiellose Mobilisierung über das soziale Medium Facebook gelungen, wie Abbildung 1 verdeutlicht. Darin ist die Anzahl der Kommentare aufgeführt, die auf die eigenen Facebook-Meldungen nur der Parteien gemacht wurden. Gemessen an diesen Nutzerreaktionen, konnte die AfD auf ihrer Facebook-Fanseite eine Aktivität hervorrufen, wie sie die vier Parteien, mit der darauffolgend höchsten Aktivität, lediglich gemeinsam erzielt haben.

Abbildung 2: Anzabl der Meldungen auf den Facebook-Fanseiten der Parteien (14-Tage-Intervalle ab 1. April 2017) $)^{55}$

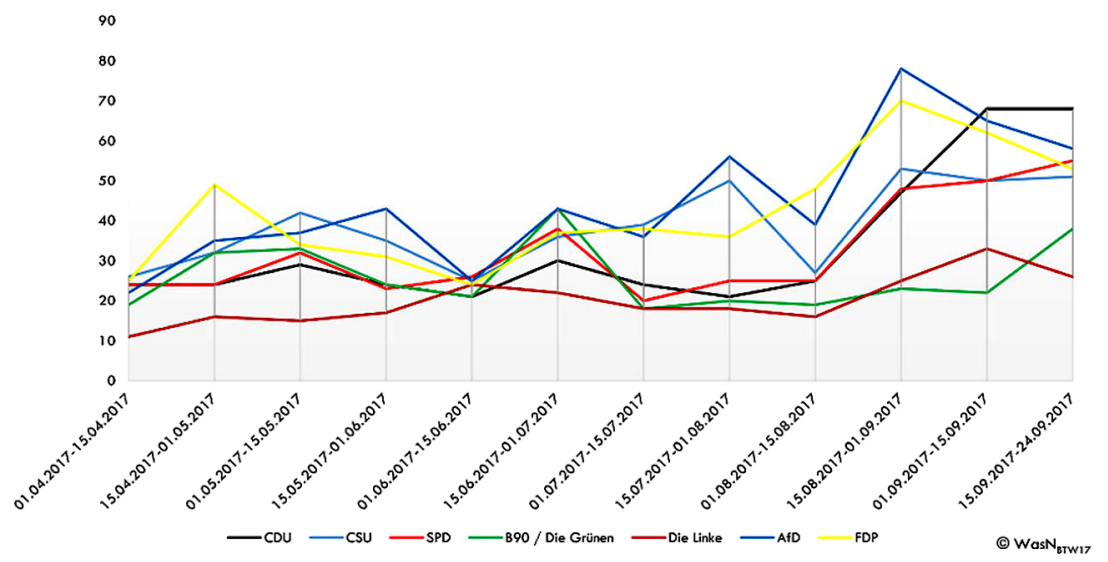

55 Quelle: eigene Darstellung. 
Abbildung 3: Anzabl der Kommentare auf den Facebook-Fanseiten der Parteien (14-Tage-Intervalle ab 1. April 2017) 56

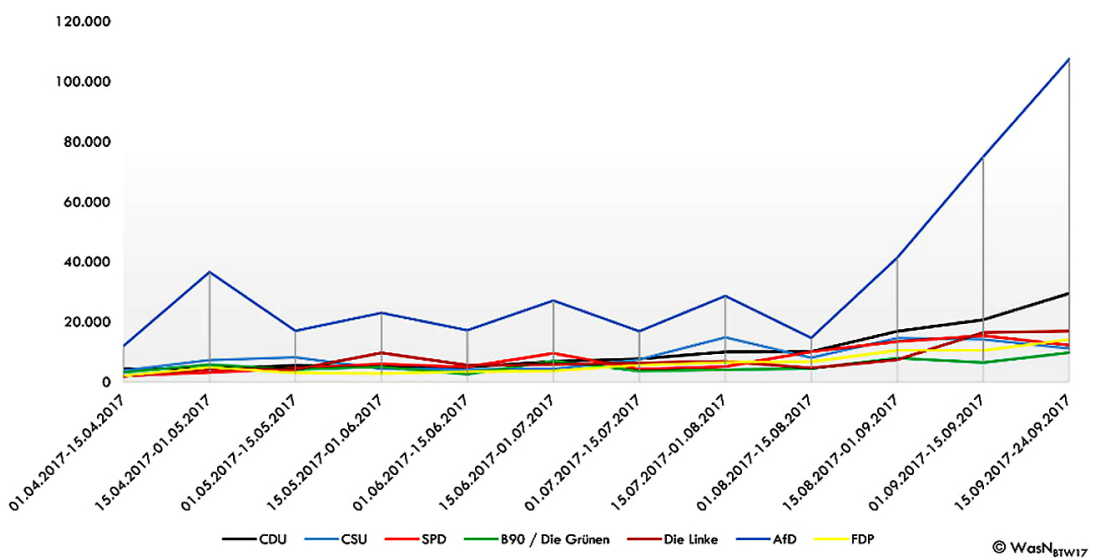

Abbildung 2 und 3 zeigen Verlaufsverteilungen der Facebook-Aktivität nach Partei über das halbe Jahr bis zur Bundestagswahl, gemessen in 14Tage-Intervallen. Im Vergleich der beiden Verläufe - Abbildung 2 zeigt die Kurve für die eigenen Meldungen (Posts) der Parteien, Abbildung 3 diejenige für die Nutzerkommentare - wird auch deutlich, dass die AfD zwar selbst durchaus ein hohes, zeitweise sogar das höchste Maß an eigener Aktivität über den gesamten Zeitraum aufweist, dass sie von den etablierten Parteien aber eingeholt (FDP) oder überholt wird (zum Schluss deutlich CDU). Betrachtet man allerdings die Nutzerreaktionen, die die Parteien durch ihre eigene Aktivität hervorrufen, dann befindet sich die AfD über den gesamten Zeitraum mit großem Abstand an der Spitze.

Doch worin zeichnet sich die besondere Aktivität der Besucherinnen und Besucher der AfD-Seite qualitativ aus? Um dies herauszufinden, haben wir für die gesammelten Kommentarbereiche der Parteien jeweils Schlüsselwörter gebildet. Hierbei handelt es sich um ein korpuslinguistisches Standardverfahren, wobei ein zu untersuchendes Teilkorpus, also etwa alle Kommentare auf der FB-Seite der AfD, mit einem sog. Referenzkorpus, bestehend aus allen Kommentaren der übrigen Parteien, verglichen wird. Ausgerechnet wird für jedes einzelne Element (also Wort), ob es signifikant häufig im Vergleich zum Referenzkorpus auftritt. Die Wörter, die die höchste Differenz aufweisen, werden als Schlüsselwörter gelistet.

56 Quelle: eigene Darstellung. 
Bereits diese einfache Schlüsselwortanalyse macht deutlich, wie sehr der Kommentarbereich auf der FB-Seite der AfD durch eine unzivilisierte Sprache im Verhältnis zu den entsprechenden Bereichen auf den Seiten der anderen Parteien geprägt ist. Tabelle 1 führt die Top-Schlüsselwörter 1-50 auf. Darunter finden sich z.B.: »Toastbrot« und »Schlampe« als beleidigende Ausdrücke für Personen; »Altparteienbande«, »Lügenpresse« und »linksversifften « als Elemente des hetzerischen Angriffs auf den politischen Gegner und die etablierten Medien sowie "Ferkel« und »Ralle« (oder Pöbel-Ralle) als Beispiele eines aggressiven Soziolekts, durch den Spitzenpolitikerinnen und Politiker wie Bundeskanzlerin Angela Merkel und das ehemalige Mitglied des SPD-Bundesvorstands Ralf Stegner regelmäßig verunglimpft und attackiert werden.

Die Einordnung zu interpretativen Kategorien erschließt sich jeweils aus dem Kontext. So seien hier für die Schlüsselwörter "Gutmenschen« und »Ferkel« jeweils eine Fundstelle wiedergegeben.

Könnte nur kotzen. Es können sich jetzt etliche Gutmenschen wie Bedford-Strohm oder Schnaps-Käsmann [sic!], die ständig von Liebe, auch Verbrechern gegenüber, faseln, entrüsten: Diese ganze Verräterbande in Berlin gehört in Ketten gelegt und für immer bei Dunkelhaft weggesperrt. (Nutzerkommentar AfD-FB-Seite)

Ferkel, Dickie, und Flintenuschi wird sicherlich keiner vermissen. Nächstes jahr ist merkel dran wenn wahlen sind. kann sich von der clinton was abschauen . was verlieren heißt. die dreht sich bestimmt jetzt im kreis und Mutti sizt [sic!] im sauerstoffzeit [sic!] wegen schnappatmung und die Grünen werden sagen das [sic! ] sie die wahl von trump nicht anerkennen. den ihre fressen möchte ich jetzt sehen. Ich freue mich schon so auf das erste treffen. (Nutzerkommentar AfD-FB-Seite)

Dies sind nur einige wenige Ausschnitte aus dem bzw. Einblicke in das Diskursverhalten auf Facebook, wie es insbesondere für den Kommentarbereich auf der FB-Seite der AfD - und hier als in besonderer Weise prägend - ausgemacht werden kann. Es gehört zur Vollständigkeit hinzugefügt, dass vergleichbare Schlüsselwortanalysen für alle anderen Parteien keine in dieser Hinsicht auffälligen Befunde gezeigt haben. Stattdessen stehen neben dem eigenen Personal durchaus Wahlkampfthemen oder thematische »Dauerbrenner« (wie etwa die Hartz-Reformen bei der SPD) im Mittelpunkt. Politische Angriffe kommen selbstverständlich vor, allerdings ist der Ton deutlich gemäßigter, und die Auseinandersetzung sachlicher als im Kommentarbereich der AfD. 
Tabelle 1: Top-50-Schlüsselwörter AfD-FB-Kommentare

\begin{tabular}{|c|c|c|}
\hline Schlüsselwörter 1-17 & $18-34$ & $35-50$ \\
\hline afdafd & aslan & onur \\
\hline weidel & toastbrot & gauland \\
\hline meuthen & schlampe & björn \\
\hline kölsch & hoeneß & vorzeit \\
\hline fehlerquoten & quickenloan69@gmail.com & wahlbeobachter \\
\hline käßmann & reizbar & altpartei \\
\hline storch & ralle & pöbel-ralle \\
\hline pretzell & presserat & altparteibande \\
\hline bülent & poggenburg & darlehen \\
\hline höcke & gutmenschen & köter \\
\hline höckes & emnid & multinationalsozialisten \\
\hline petry & lügenpresse & altparteien \\
\hline frauke & bundesverdienstkreuz & lucke \\
\hline ferkel & driesang & linksversifften \\
\hline afdgrüße & beatrix & bubu \\
\hline alice & transaktion & reker \\
\hline reil & religionsideologie & \\
\hline \multicolumn{3}{|c|}{ Legende: } \\
\hline $\begin{array}{c}\text { Parteiname/ } \\
\text { Kampagnenformel }\end{array}$ & $\begin{array}{c}\text { eigene Akteure/ } \\
\text { Parteipolitiker/-innen }\end{array}$ & $\begin{array}{c}\text { Beleidigung/ } \\
\text { aggressiver Soziolekt }\end{array}$ \\
\hline
\end{tabular}

\section{Fazit: Schattenseite der Online-Partizipation oder diskursive Echokammer}

Was bedeuten die Befunde mit Blick auf die übergeordnete Frage, ob also die sozialen Medien das demokratische Diskursverhalten verändern? Zunächst einmal ist festzuhalten, dass das unzivilisierte Verhalten in unserem Datensatz zur Facebook-Kommunikation im deutschen Bundestagswahlkampf gleichsam einen Namen hat. Es heißt AfD, insofern als wir die gesuchten Phänomene der Hassrede und Pöbelei überwiegend im entsprechenden Teilkorpus für die AfD finden, und weit weniger bei den anderen Parteien. Eine auf Nutzernetzwerken basierende Studie der Süddeutschen 
Zeitung hatte bereits im Vorfeld der Bundestagswahl (Mai 2017) gezeigt, ${ }^{57}$ dass es in Deutschland grundsätzlich keine Echokammern in der politischen Diskussion gebe, wohl aber für die AfD. In diese Richtung weisen auch die Befunde zur Verrohung der Sprache in den Facebook-Kommentaren, wie sie hier schlaglichtartig präsentiert worden sind.

Dieser Befund kann, so er sich denn erhärten lässt, zweierlei bedeuten: Es sind nicht die sozialen Netzwerke allgemein, die sich negativ auf unsere politische Kommunikation auswirken. Das Bedürfnis zu beleidigen, auszugrenzen, das empfundene Korsett politischer Korrektheit auf drastische Weise zu durchbrechen, geht von den Nutzerinnen und Nutzern aus und wird von politischen Bewegungen und Parteien wie der AfD offenbar in besonderer Weise bedient. Das Internet und soziale Netzwerke ermöglichen ihnen - insoweit haben Buchstein und andere Kritiker Recht -, eine Parallelöffentlichkeit, eine eigene Öffentlichkeit ohne die eingeübten zivilisatorischen oder redaktionellen Filter zu nutzen, um ihren privaten Regungen öffentlich Ausdruck zu verleihen.

Um die Effekte auf die Demokratie abschätzen zu können, wird es darauf ankommen, welche Spill-over-Effekte dieses Diskursverhalten auf andere Communities, andere Plattformen, den Offline-Diskurs und die politische Debatte insgesamt hat. Dass wir die Verrohung der Sprache noch klar zuschreiben können, kann nicht bedeuten, dass sich die Mehrheitsgesellschaften zurücklehnen und auf die Selbstdemontage der AfD und ähnlicher Kräfte in der parlamentarischen Arbeit warten können. Zum einen haben die Rechtspopulisten in Deutschland und anderswo kräftige Polster parlamentarischer Repräsentation anhäufen können. Doch wie lassen sich gewisse Schutzmauern einer wehrhaften Demokratie gegen die Verrohung im demokratischen Diskurs hochziehen? Die Gemeinschaftsstandards der Anbieter werden kaum ausreichen, um den Hetzern und Kämpfern gegen die politische Korrektheit den Boden zu entziehen, abgesehen davon, dass diese vielfach intransparent sind, mangelhaft durchgesetzt werden und legitimatorisch problematisch sind. Umgekehrt laufen gesetzliche Regelungen wie das deutsche Netzwerkdurchsetzungsgesetz Gefahr, entweder ineffektiv zu sein (denn auf Ko-Regulierung der Unternehmen sowie tatsächliche Strafbarkeit der Inhalte angewiesen) oder grundlegende liberaldemokratische Normen zu verletzen.

57 Jannis Brühl, Katharina Brunner und Sabrina Ebitsch, »Der Facebook-Faktor: Wie das soziale Netzwerk die Wahl beeinflusst", Süddeutsche Zeitung (München, 2017) http://gfx.sueddeutsche.de/apps/e502288/www/ (abgerufen am 4. Februar 2018). 
All dies geht mit einem deutlichen Wandel in den Bewertungen des Internets und seines Effekts auf die Demokratieentwicklung einher. In der öffentlichen und politischen Diskussion ist auf die große Euphorie große Ernüchterung gefolgt. Die Rede von dem Internet als Befreiungstechnologie und Demokratieförderer geht heute nur noch wenigen von den Lippen. Vielmehr erscheinen Internet und Digitalisierung heute vermehrt als Gefährdung der Demokratie. Die Hochgesänge auf die Entmachtung der Gatekeeper und die Ertüchtigung des Individuums kehren als geradezu bedrohlich verzerrtes Echo zurück. Aus der freudvollen Stimmung des "Here comes everybody "wird aktuell ein - wenn auch bislang in demokratischen Räumen nur hinter vorgehaltener Hand hörbares - »Wehe, wenn sie losgelassen«. In jedem Fall müssen sich die demokratischen Gesellschaften fragen, wie sie auf die Verrohung politischer Diskurse reagieren und insbesondere deren mögliche Effekte auf die politische Debatte. 
